\title{
Edge Vertex Prime Labeling of Union of Graphs
}

\section{Simaringa, S. Muthukumaran}

Abstract: A graph $G(p, q)$ is said to be an edge vertex prime labeling if its vertices and edges are labeled with distinct positive numbers not exceeding $p+q$ such that for any edge $e=x y_{x}$ $f(x), f(y)$ and $f(x y)$ are pairwise relatively prime. We prove that some class of union of graphs such as $p+q$ is even for $\boldsymbol{G} \cup K_{1, n}, \boldsymbol{G} \cup P_{n}$ and $\boldsymbol{C}_{m} \cup K_{1, n}, \boldsymbol{C}_{m} \cup \boldsymbol{P}_{n}, \boldsymbol{C}_{\boldsymbol{n}} \cup \boldsymbol{C}_{\boldsymbol{n}}$ when $\boldsymbol{n} \equiv \mathbf{0}, 2(\bmod 3), K_{2_{i} m} \cup \boldsymbol{C}_{n}$ and one point union of wheel and cycle related graphs are edge vertex prime.

Keywords: edge vertex prime labeling, relatively prime, star, path, cycle, one point union of graphs.

Mathematics Subject Classification: 05C78

\section{INTRODUCTION}

Finite, simple and undirected graphs should alone be considered. A graph $G$ is an ordered pair $G=(V, E)_{3}$ where $V(G)$ refer a finite set of elements called vertices, while $E(G)$ is a finite set of unordered pairs of vertices called edges. The cardinality of the sets of vertices $V(G)$ and edges $E(G)$ is denoted by $|V(G)|$ and $|E(G)|$ respectively. For all standard notation and terminology in graph theory, Balakrishnan and Ranganathan [1] are followed. A graph of order $n$ is prime if one can bijectively label its vertices with positive numbers $1,2,3, \ldots, n$, so that any two adjacent vertices are relatively prime. Tout, Dobboucy, Howalla [10], first introduced a first kind of graph labeling called prime labeling and later developed by Roger Entriger. There are several types of labeling for a dynamic survey of various graph labeling problems with extensive bibliography we refer to Gallian [2]. Let $G_{1}=\left(V_{1}, E_{1}\right)$ and $G_{2}=\left(V_{2}, E_{2}\right)$ be two simple graphs. The graph $G=(V(G), E(G)), \quad$ where $\quad V=V_{1} \cup V_{2} \quad$ and $E=E_{1} \cup E_{2}$ is called the union of $G_{1}$ and $G_{2}$ is denoted by $G_{1} \cup G_{2}$. For $n \geq 2$ an $n$ - path or simply path is denoted $P_{n}$ is a connected graph consisting of two vertices, with degree 1 and $n-2$ vertices of

degree 2. For $n \geq 3$, an $n$-Cycle or Simply cycle, denoted $C_{n}$, is a connected graph

Revised Manuscript Received on December 15, 2019

M. Simaringa, Department of Mathematics, Thiru Kolanjiappar Govt. Arts College,, Virudhachalam, Tamilnadu, India.

Email: simaringalancia@gmail.com

S. Muthukumaran, Department of Mathematics, Siga College of Management and Computer science, Villupuram, Tamilnadu, India.

Email:smuthukumaranmaths@gmail.com consisting of $n$ vertices, each of degree 2. Note that both $P_{n}$ and $C_{n}$ have $n$ vertices while $P_{n}$ has $n-1$ edges and $C_{n}$ has $\mathrm{n}$ edges. An $n-$ star or simply star, denoted $S_{n}$ is a graph consisting of one vertex of degree $n_{p}$ called the centre and $n$ vertices of degree 1 . Note that $S_{n}$ consists of $n+1$ vertices and $n$ edges. The graph $W_{n}^{m}$ obtained from $m$ copies of $W_{n}$ by identifying their center. Prime labeling is a variant of an edge vertex prime labeling. An edge vertex prime labeling starts with the definion of a bijective function $f: V(G) \cup E(G) \rightarrow\{1,2,3, \ldots,|V(G) \cup E(G)|\}$ is an edge vertex prime labeling if for any edge $u v \in E(G)$, we have

$$
\begin{aligned}
& \operatorname{gcd}(f(u), f(v))=\operatorname{gcd}(f(u), f(u v))= \\
& \operatorname{gcd}(f(v), f(u v))=1
\end{aligned}
$$

. A graph $G$ which admits an edge vertex prime labeling is called an edge vertex prime graph. Jagadesh and Baskar Babujee [3] originated the concept of an edge vertex prime labeling the existence of the same paths, cycles and star $K_{1, n}$ are proved by them. In [4], they also proved that an edge vertex prime graph for some class of graphs such as generalized star, generalized cycle star, $p+q$ is even for $G \widehat{O} K_{1, n}, G \widehat{O} P_{n}, G \widehat{O} C_{n}$ * An edge vertex prime graph of wheel graph, fan graph, friendship graph is Parmer [5] investigated. An edge vertex prime graph can be [6] determined that $K_{2, n}$ for every $n$ and $K_{3, n}$ for $n=\{3,4, \ldots, 29\}$.

In [7], we proved that triangular and rectangular book, butterfly graph with shell, Drums $D_{n}$, Jahangir $I_{n, 3^{x}}$ and $I_{n, 4}$ are an edge vertex prime graphs. Also in [8], double star $B_{m, n}$, subdivision of $B_{m, n}$ and $K_{1, n}$, comb graph, spider, H-graph of path $P_{n}$ and coconut tree are an edge vertex prime graph are determined by us. Some class of graphs such as $p+q$ is odd for $G \widehat{O O} W_{n}, G \widehat{O} f_{n}, G \widehat{O} F_{n}$, $p+q$ is even for $G \widehat{O} P_{n}, C_{l} \widehat{O} K_{1, m} \widehat{O} P_{n}$, Umbrella graph $U(m, n)$, crown graph, union of cycles for $C_{n} \cup C_{n} \cup C_{n}$ $n \equiv 0(\bmod 3) \quad, \quad C_{n} \cup C_{n} \cup C_{n} \cup \ldots \cup C_{n}$ $n \equiv 0(\bmod 5)$ are an edge vertex prime graph. 
In section 2. An edge vertex prime is an investigation of union of some graphs $p+q$ is even for $G \cup K_{1, n}$ $G \cup P_{n}$, and $C_{m} \cup K_{1_{n},}, \quad C_{m} \cup P_{n}, \quad C_{n} \cup C_{n}$ when $n \equiv 0,2(\bmod 3)$

$K_{2, m} \cup C_{n}$

when $m$ is even, $n \equiv 0(\bmod 3)$ and $m$ is odd $n \equiv 0,1(\bmod 3)$.

In section 3, finalise that one point union of graphs such as $W_{n}^{m}, n$ is even and $n=3,5,7,9$ and cycle $C_{n}^{m}$, $n=3,4,5,6,7,9,11$ are an edge vertex prime.

\section{UNION OF GRAPHS}

We have proved some union of graphs are edge vertex prime in the section below.

Theorem 2.1 If $G(p, q)$ has an edge vertex prime graph with $p+q$ is even, then there exists a graph from the class $G \cup K_{1, n}, n \geq 1$ that admits an edge vertex prime graph.

Proof. Let $G(p, q)$ be an edge vertex prime graph when $p+q$ is even, with bijective function $f: V(G) \cup E(G) \rightarrow\{1,2, \ldots, p+q\}$ with property that given any edge $u v \in E(G)$, the numbers $f(u), f(v)$ and $f(u v)$ are pairwise relatively prime. Consider the graph $K_{1, n}$ with vertex set $\left\{u, v_{i}: 1 \leq i \leq n\right\}$ and edge set $\left\{u v_{i}: 1 \leq i \leq n\right\}$. We define a new graph $G_{1}=G \cup K_{1, n} \quad$ with vertex set $V_{1}=V(G) \bigcup\left\{u, v_{i}: 1 \leq i \leq n\right\} \quad$ and edge set $E_{1}=E \bigcup\left\{u v_{i}: 1 \leq i \leq n\right\}$. Define a bijective function $g: V_{1} \cup E_{1} \rightarrow\{1,2,3, \ldots, p+q, p+q+1, \ldots, p+$ $q+2 n+1\}$

by $g(v)=f(v) \quad, \quad$ for all $v \in V(G)$ and $g(u v)=f(u v)$ for all $u v \in E(G), g(u)=p$, where $p$ is choose the largest prime number in the set $\{p+q+1, p+q+2, \ldots, p+q+2 n+1\} \quad$ and label the edge set $\left\{u v_{i}: 1 \leq i \leq n\right\}$ by remaining even labels and label the vertex set $\left\{v_{i}: 1 \leq i \leq n\right\}$ by the remaining odd labels. To analyse that $G_{1}$ is an edge vertex prime graph. Earlier, $G$ is an edge vertex prime graph, it is possible to prove that for any edge $u v \in E_{1}$, which is not in $G_{y}$ the numbers $g(u), g(v)$ and $g(u v)$ are pairwise relatively prime. It is easily proved that, for any edge $u v \in E_{1}$ $\operatorname{gcd}(g(u), g(v))=1$ $\operatorname{gcd}(g(u), g(u v))=1, \operatorname{gcd}(g(v), g(u v))=1$. Hence $G_{1}=G \cup K_{1_{2} n}, n \geq 1$ is an edge vertex prime graph.
Theorem 2.2 If $G(p, q)$ has an edge vertex prime graph with $p+q$ is even, then there exists a graph from the class $G \bigcup P_{n}$ that admits an edge vertex prime graph.

Proof. Let $G(p, q)$ be an edge vertex prime labeling graph when $p+q$ is even, with bijective function $f: V(G) \cup E(G) \rightarrow\{1,2, \ldots, p+q\}$ with property that given any edge $u v \in E(G)$, the numbers $f(u), f(v)$ and $f(u v)$ are pairwise relatively prime. Consider the graph $P_{n}$ with vertex set $\left\{u_{i}: 1 \leq i \leq n\right\}$ and edge set $\left\{u_{i} u_{i+1}: 1 \leq i \leq n-1\right\}$. We define a new graph $G_{1}=G \bigcup P_{n}$ with vertex set $V_{1}=V \bigcup\left\{u_{i}: 1 \leq i \leq n\right\}$ and $E_{1}=E \bigcup\left\{u_{i} u_{i+1}: 1 \leq i \leq n-1\right\}$. Define a bijective

function

$g: V_{1} \cup E_{1} \rightarrow\{1,2,3, \ldots, p+q, p+q+1, \ldots, p+$ $q+2 n-1\}$

by $g(v)=f(v) \quad$ for all $\quad v \in V(G) \quad$ and $g(u v)=f(u v) \quad$ for $\quad$ all $\quad u v \in E(G)$, $g\left(u_{i}\right)=p+q-1+2 i \quad$ for $\quad 1 \leq i \leq n_{2}$ $g\left(u_{i} u_{i+1}\right)=p+q+2 i$ for $1 \leq i \leq n-1$. We have to prove that $G_{1}$ is an edge vertex prime labeling. Earlier, $G$ is an edge vertex prime labeling, it is enough to prove that for any edge $u v \in E_{1}$, which is not in $G_{s}$ the numbers $g(u), g(v)$ and $g(u v)$ are pairwise relatively prime. Label the vertices and edges of path $P_{n}$ is consecutive positive numbers. It is easily verified that, for any edge $\in E_{1}$ $\operatorname{gcd}(g(u), g(v))=1$ $\operatorname{gcd}(g(u), g(u v))=1, \operatorname{gcd}(g(v), g(u v))=1$ So $G_{1}=G \bigcup P_{n}$ is an edge vertex prime graph.

Theorem 2.3 The disconnected graph $C_{m} \cup K_{1, n}, m \geq 3$ is an edge vertex prime graph.

Proof. Consider the disconnected graph $G=C_{m} \cup K_{1, n^{*}}$ Let $\quad V\left(c_{m}\right)=\left\{v_{i}: 1 \leq i \leq m\right\} \quad$ and $V\left(K_{1, n}\right)=\left\{u_{,} u_{i}: 1 \leq i \leq n\right\}$, where $u$ is the centre of $K_{1, n}, \quad E\left(C_{m}\right)=\left\{v_{1} v_{m}, v_{i} v_{i+1}: 1 \leq i \leq m-1\right\}$, $E\left(K_{1, n}\right)=\left\{u u_{i}: 1 \leq i \leq n\right\}$,

Also,

$|V(G)|=m+n+1$ and $|E(G)|=m+n$. Define a bijective function

$f: V(G) \cup E(G) \rightarrow\{1,2, \ldots, 2 m+2 n+1\}$ by $f(u)=1 \quad, \quad f\left(u_{i}\right)=2 i+1 \quad$ for $\quad 1 \leq i \leq n_{2}$ $f\left(u u_{i}\right)=2 i \quad$ for $\quad 1 \leq i \leq n_{2}$ $f\left(v_{1}\right)=2 m+2 n+1_{y} \quad f\left(v_{i}\right)=2 n+2 i-1$ for $2 \leq i \leq m_{p}$ $f\left(v_{i} v_{i+1}\right)=2 n+2 i$ for

$1 \leq i \leq m-1$,

$f\left(v_{1} v_{m}\right)=2 m+2 n$. 
Next, we show that the property of an edge vertex prime graph.

For any $1 \leq i \leq n_{\text {, }}$

$\operatorname{gcd}\left(f(u), f\left(u_{i}\right)\right)=\operatorname{gcd}(1,2 i+1)=1$,

$\operatorname{gcd}\left(f(u), f\left(u u_{i}\right)\right)=\operatorname{gcd}(1,2 i)=1$,

$\operatorname{gcd}\left(f\left(u_{i}\right), f\left(u u_{i}\right)\right)=\operatorname{gcd}(2 i+1,2 i)=1$, since

they are consecutive positive numbers. For any $2 \leq i \leq n$, $\operatorname{gcd}\left(f\left(v_{i}\right), f\left(v_{i+1}\right)\right)=\operatorname{gcd}(2 n+2 i-1,2 n+$ $2 i+1)=1$,

$\operatorname{gcd}\left(f\left(v_{i}\right), f\left(v_{i} v_{i+1}\right)\right)=\operatorname{gcd}(2 n+2 i-1,2 n+$ 2i) $=1$,

$\operatorname{gcd}\left(f\left(v_{i+1}\right), f\left(v_{i} v_{i+1}\right)\right)=\operatorname{gcd}(2 n+2 i+$ $1,2 n+2 i)=1$,

$\operatorname{gcd}\left(f\left(v_{1}\right), f\left(v_{2}\right)\right)=\operatorname{gcd}(2 m+2 n+1,2 n+$ 3) $=1$,

$\operatorname{gcd}\left(f\left(v_{1}\right), f\left(v_{1} v_{2}\right)\right)=\operatorname{gcd}(2 m+2 n+1,2 n+$ 2) $=1$,

$\operatorname{gcd}\left(f\left(v_{2}\right), f\left(v_{1} v_{2}\right)\right)=\operatorname{gcd}(2 n+3,2 n+2)=$ 1 ,

$\operatorname{gcd}\left(f\left(v_{1}\right), f\left(v_{m}\right)=\operatorname{gcd}(2 m+2 n+1,2 m+2 n-\right.$ $\operatorname{gcd}\left(f\left(v_{1}\right), f\left(v_{1} v_{m}\right)\right)=\operatorname{gcd}(2 m+2 n+1,2 m+$ $\operatorname{gcd}\left(f\left(v_{m}\right), f\left(v_{1} v_{m}\right)\right)=\operatorname{gcd}(2 m+2 n-$ $1,2 m+2 n)=1$.

Therefore, for any edge $\in E(G)$, $\operatorname{gcd}(f(u), f(v))=1, \operatorname{gcd}(f(u), f(u v))=1$, $\operatorname{gcd}(f(v), f(u v))=1$

Hence $G=C_{m} \cup K_{1, n}, m \geq 3$ admits an edge vertex prime graph.

Theorem 2.4 The disconnected graph $C_{m} \cup P_{n}, m \geq 3$ is an edge vertex prime graph.

Proof. Let $u_{1}, u_{2}, \ldots, u_{m}$ be the vertices of cycle $C_{m}$ and $v_{1}, v_{2}, \ldots, v_{n}$ be the vertices of path $P_{n^{*}}$ Consider $G=C_{m} \cup P_{n} \quad$ be $\quad$ a graph. Then $V(G)=\left(u_{i}, v_{j}: 1 \leq i \leq m \quad, \quad 1 \leq j \leq n\right\} \quad$ and $E(G)=\left\{u_{1} u_{m}, u_{i} u_{i+1}: 1 \leq i \leq m-\right.$

1) $\left\{v_{j} v_{j+1}: 1 \leq i \leq n-1\right\}$.

Here, $|V(G)|=m+n$ and $|E(G)|=m+n-1$. Define a bijective function $f: V(G) \cup E(G) \rightarrow\{1,2, \ldots, 2 m+2 n-1\} \quad$ by $f\left(u_{i}\right)=2 i-1$ for $1 \leq i \leq m, f\left(u_{i} u_{i+1}\right)=2 i$ for y
$1 \leq i \leq m-1$,

$f\left(v_{j}\right)=2 m+2 j-1$ for

$$
f\left(u_{1} u_{m}\right)=2 m
$$

$f\left(v_{j} v_{j+1}\right)=2 m+2 j$ for $1 \leq j \leq n-1$.

Next, we prove the property of an edge vertex prime graph.

For any edge $u_{i} u_{i+1} \in E(G)$,

$\operatorname{gcd}\left(f\left(u_{i}\right), f\left(u_{i+1}\right)\right)=\operatorname{gcd}(2 i-1,2 i+1)=1$, $\operatorname{gcd}\left(f\left(u_{i}\right), f\left(u_{i} u_{i+1}\right)\right)=\operatorname{gcd}(2 i-1,2 i)=1$,

$\operatorname{gcd}\left(f\left(u_{i+1}\right), f\left(u_{i} u_{i+1}\right)\right)=\operatorname{gcd}(2 i+1,2 i)=1$.

For any $u_{1} u_{m} \in E(G)$,

$\operatorname{gcd}\left(f\left(u_{1}\right), f\left(u_{m}\right)\right)=\operatorname{gcd}(1,2 m-1)=1$,

$\operatorname{gcd}\left(f\left(u_{1}\right), f\left(u_{1} u_{m}\right)\right)=\operatorname{gcd}(1,2 m)=1$,

$\operatorname{gcd}\left(f\left(u_{m}\right), f\left(u_{1} u_{m}\right)\right)=\operatorname{gcd}(2 m-1,2 m)=1$.

Similarly, the other edges are pairwise relatively prime. Therefore, for any edge $u v \in E(G)$, $\operatorname{gcd}(f(u), f(v))=1, \operatorname{gcd}(f(u), f(u v))=1$, $\operatorname{gcd}(f(v), f(u v))=1$. Hence $C_{m} U P_{n}, m \geq 3$ has an edge vertex prime graph.

Theorem 2.5 The disconnected graph $C_{n} \cup C_{n}, n \geq 3$ admits an edge vertex prime graph, where $n \equiv 0,2(\bmod 3)$.

Proof. Let $G=C_{n} \cup C_{n}$ be a graph. Then $V(G)=\left\{v_{i}: 1 \leq i \leq 2 n\right\}$

and

$E^{\prime}(G) \stackrel{1}{=}\left\{v_{i} v_{i+1}: 1 \leq i \leq n-\right.$

1 )

1) $U\left\{v_{n+1} v_{2 n}\right\}$.

Also, $|V(G)|=2 n$ and $|E(G)|=2 n$. Define a bijective function $f: V(G) \cup E(G) \rightarrow\{1,2, \ldots, 4 n\}$ by $f\left(v_{i}\right)=2 i-1$ for $1 \leq i \leq 2 n, f\left(v_{i} v_{i+1}\right)=2 i$ for $1 \leq i \leq n-1, f\left(v_{1} v_{n}\right)=2 n, f\left(v_{n+1} v_{2 n}\right)=4 n$, $f\left(v_{i} v_{i+1}\right)=2 i$ for $n+1 \leq i \leq 2 n-1$. Clearly, for any edge $u v \in E(G), \quad \operatorname{gcd}(f(u), f(v))=1$, $\operatorname{gcd}(f(u), f(u v))=1, \operatorname{gcd}(f(v), f(u v))=1$ Hence $G=C_{n} \cup C_{n}, n \geq 3$ is an edge vertex prime graph, where $n \equiv 0,2(\bmod 3)$.

Theorem 2.6 The graph obtained by the duplication of vertex $v_{2}$ in path $P_{n}$ or cycle $C_{n}$ is an edge vertex prime graph.

Proof. Let $G^{b}$ be the graph obtained by duplicating a vertex $v_{2}$ of degree 2 in $P_{n^{x}}$ Let $v_{2}^{J}$ be the duplication of $v_{2}$ in $G^{\prime}$. Then $V\left(G^{\prime}\right)=\left\{v_{2}^{v}, v_{i}: 1 \leq i \leq n\right\}$ and $E\left(G^{\prime}\right)=\left\{v_{i} v_{i+1}: 1 \leq i \leq n-\right.$ 1\} $\cup\left\{v_{1} v_{2}^{\prime}\right\} \cup\left\{v_{3} v_{2}^{\prime}\right\}$. 
Here, $\left|V\left(G^{\prime}\right)\right|=n+1$ and $\left|E\left(G^{\prime}\right)\right|=n+1$. Define a bijective

$f: V\left(G^{\prime}\right) \cup E\left(G^{\prime}\right) \rightarrow\{1,2, \ldots, 2 n+2\}$ labeling

$f\left(v_{i}\right)=2 i-1$ for $1 \leq i \leq n, f\left(v_{i} v_{i+1}\right)=2 i$ for $1 \leq i \leq n-1, \quad f\left(v_{2}^{\prime}\right)=2 n+1, f\left(v_{1} v_{2}^{\prime}\right)=2 n$, $f\left(v_{3} v_{2}^{\prime}\right)=2 n+2$.

Next, we show that the property of an edge vertex prime graph.

For any $1 \leq i \leq n-1$,

$\operatorname{gcd}\left(f\left(v_{i}\right), f\left(v_{i+1}\right)\right)=\operatorname{gcd}(2 i-1,2 i+1)=1$, $\operatorname{gcd}\left(f\left(v_{i}\right), f\left(v_{i} v_{i+1}\right)\right)=\operatorname{gcd}(2 i-1,2 i)=1$, $\operatorname{gcd}\left(f\left(v_{i+1}\right), f\left(v_{i} v_{i+1}\right)\right)=\operatorname{gcd}(2 i+1,2 i)=1$, $\operatorname{gcd}\left(f\left(v_{1}\right), f\left(v_{2}^{\prime}\right)\right)=\operatorname{gcd}(1,2 n+1)=1$, $\operatorname{gcd}\left(f\left(v_{1}\right), f\left(v_{1} v_{2}^{\prime}\right)\right)=\operatorname{gcd}(1,2 n)=1$, $\operatorname{gcd}\left(f\left(v_{2}^{\prime}\right), f\left(v_{1} v_{2}^{\prime}\right)\right)=\operatorname{gcd}(2 n+1,2 n)=1$, $\operatorname{gcd}\left(f\left(v_{3}\right), f\left(v_{2}^{\prime}\right)\right)=\operatorname{gcd}(5,2 n+1)=1$, $\operatorname{gcd}\left(f\left(v_{3}\right), f\left(v_{3} v_{2}^{\prime}\right)\right)=\operatorname{gcd}(5,2 n+2)=1$, $\operatorname{gcd}\left(f\left(v_{2}^{\prime}\right), f\left(v_{3} v_{2}^{\prime}\right)\right)=\operatorname{gcd}(2 n+1,2 n+2)=1$. Therefore, for any edge $\in E(G)$, $\operatorname{gcd}(f(u), f(v))=1, \operatorname{gcd}(f(u), f(u v))=1$, $\operatorname{gcd}(f(v), f(u v))=1$. Hence the graph $G^{\prime}$ is duplicating a vertex $v_{2}$ in $P_{n}$ has an edge vertex prime labeling.

Let $G^{\prime \prime}$ be the graph obtained by duplication of $v_{2}$ of degree 2 in $C_{n}$. Let $v_{2}^{n t}$ be the duplication of $v_{2}$ in $G^{n}$. Then $V\left(G^{n}\right)=\left\{v_{2}^{n}, v_{i}: 1 \leq i \leq n\right\}$, and $E\left(G^{\prime \prime}\right)=\left\{v_{i} v_{i+1}: 1 \leq i \leq n-\right.$

1\}U $\left\{v_{1} v_{n}\right\} \bigcup\left\{v_{1} v_{2}^{\prime \prime}\right\} \bigcup\left\{v_{3} v_{2}^{\prime \prime}\right\}$.

Here, $V\left(G^{\prime \prime}\right)=n+1$ and $E\left(G^{\prime \prime}\right)=n+2$. Define a bijective labeling $f: V\left(G^{\prime \prime}\right) \cup E\left(G^{\prime \prime}\right) \rightarrow\{1,2, \ldots, 2 n+3\}$ by $f\left(v_{i}\right)=2 i-1$ for $1 \leq i \leq n, f\left(v_{i} v_{i+1}\right)=2 i$ for $1 \leq i \leq n-1, f\left(v_{1} v_{n}\right)=2 n, f\left(v_{2}^{\prime \prime}\right)=2 n+2$, $f\left(v_{1} v_{2}^{\prime \prime}\right)=2 n+1, f\left(v_{3} v_{2}^{\prime \prime}\right)=2 n+3$. Clearly, for any edge $u v \in E(G), \quad \operatorname{gcd}(f(u), f(v))=1$, $\operatorname{gcd}(f(u), f(u v))=1, \operatorname{gcd}(f(v), f(u v))=1$. Hence the graph $G^{n}$ is duplication of $v_{2}$ in $C_{n}$ has an edge vertex prime graph.

Theorem 2.7 The disconnected graph $K_{2, m} \cup C_{n}$, $(n \geq 3, n \equiv 0(\bmod 3), m$ is even $)$ is an edge vertex prime graph.

Proof. Consider the disconnected graph $G=K_{2, m} \cup C_{n}$, ( $n \geq 3, n \equiv 0(\bmod 3)$, $m$ is even ). Let $V\left(K_{2, m}\right)=\left\{u_{1}, u_{2}\right\} \cup\left\{v_{i}: 1 \leq i \leq m\right\}$

$V\left(C_{n}\right)=\left\{w_{i}: 1 \leq i \leq n\right\}$
$E\left(K_{2, m}\right)=\left\{u_{1} v_{i}, u_{2} v_{i}: 1 \leq i \leq m\right.$

$E\left(C_{n}\right)=\left\{w_{1} w_{n}, w_{i} w_{i+1}: 1 \leq i \leq n-1\right\}$. Also, $|V(G)|=m+n+2$ and $|E(G)|=2 m+n$. a bijective function $f: V(G) \cup E(G) \rightarrow\{1,2, \ldots, 3 m+2 n+2\}$ as follows. First, consider $K_{2, m}$, we use (Parmer [6] proved that the same technique $K_{2, m}$ is an edge vertex prime graph for all $m$ in theorem 2.1). Next, consider $C_{n}$, $f\left(w_{i}\right)=3 m+2 i+1 \quad$ for $\quad 1 \leq i \leq n$ $f\left(w_{i} w_{i+1}\right)=3 m+2 i+2$ for $1 \leq i \leq n-1$.

Clearly, for any edge $u v \in E(G)$, the numbers $f(u), f(v)$ and $f(u v)$ are pairwise relatively prime. Hence $G=K_{2, m} \cup C_{n}$

$(n \geq 3, n \equiv 0(\bmod 3)$, $m$ is even $)$ admits an edge vertex prime graph.

Theorem 2.8 The disconnected graph $K_{2, m} \cup C_{n}$, $(n \geq 3, n \equiv 0,1(\bmod 3), m$ is odd $)$ is an edge vertex prime graph.

Proof. Similar to the even case, above theorem 2.7, only changes in first cycle, we stated the lowest label by an edge. $f\left(w_{i}\right)=3 m+2 i$ for $2 \leq i \leq n$,

$f\left(w_{1}\right)=3 m+2 n+2$

$f\left(w_{i} w_{i+1}\right)=3 m+2 i+1$ for $1 \leq i \leq n-1$,

$f\left(w_{1} w_{n}\right)=3 m+2 i+1$. It is easily verified that, for any edge $u v \in E(G)$, the numbers $f(u), f(v)$ and $f(u v)$ are pairwise relatively prime.

Hence $\quad G=K_{2, m} \cup C_{n}$

$(n \geq 3, n \equiv 0,1(\bmod 3)$, $m$ is even $)$ admits an edge vertex prime graph.

\section{ONE POINT UNION OF GRAPHS}

In this section, we investigate one point union of some graphs are an edge vertex prime.

Theorem 3.1 One point union of $m$ copies $W_{n}$, that is, $W_{n}^{m}$ $(n$ is even, except $n=10 n-6,10 n-2, m \geq 1$ and $n \geq 1$ ) is an edge vertex prime graph.

Proof. Let $G=W_{n}^{m}$ be a graph. Then $V(G)=\left\{v, v_{i j}: 1 \leq i \leq m, 1 \leq j \leq n\right\}$ and $E(G)=\left\{v v_{i j}: 1 \leq i \leq m, 1 \leq j \leq n\right\} \quad \cup$ $\left\{v_{i j} v_{i j+1}: 1 \leq i \leq m, 1 \leq j \leq n-1\right\} \mathrm{U}$

$$
\left\{v_{i 1} v_{i n}: 1 \leq i \leq m\right\}
$$

Also,

$|V(G)|=m n+1$ and $|E(G)|=2 m n$. Define a bijective function $f: V(G) \cup E(G) \rightarrow\{1,2, \ldots, 3 m n+1\}$ by 
$f\left(v_{i j}\right)=\left\{\begin{array}{lc}3 n(i-1)+3 j ; & j=1,3,5, \ldots, n-1 f\left(v_{i 1} v_{i j}\right)=3(10 n-6)(i-1)+3 j+1, j= \\ 3 n(i-1)+3 j-1 ; & j=2,4,6, \ldots, n 10 n-6\end{array}\right.$

$f\left(v v_{i j}\right)=$

$\left\{\begin{array}{l}3 n(i-1)+3 j-1 ; \\ 3 n(i-1)+3 j\end{array}\right.$

$j=1,3,5, \ldots, n-1$
$j=2,4,6, \ldots, n$

$f\left(v_{i j} v_{i j+1}\right)=3 n(i-1)+3 j+1, j=$

$1,3,5, \ldots, n-1$

$$
f\left(v_{i 1} v_{i j}\right)=3 n(i-1)+3 j+1, j=n .
$$

It is easily verified that, for any edge $u v \in E(G)$, $\operatorname{gcd}(f(u), f(v))=1, \operatorname{gcd}(f(u), f(u v))=1$, $\operatorname{gcd}(f(v), f(u v))=1$. Hence $G=W_{n}^{m}$ ( $n$ is even, except $n=10 n-6,10 n-2, m \geq 1$ and $n \geq 1$ ) admits an edge vertex prime graph.

Theorem 3.2 One point union of $W_{10 n-6}^{m}$, $m \geq 1$ and $n \geq 1$ is an edge vertex prime graph.

Proof. Let $G=W_{10 n-6}^{m}$ be a graph. Then $V(G)=\left\{v, v_{i j}: 1 \leq i \leq m, 1 \leq j \leq\right.$ $10 n-6\}$

and

$$
\begin{array}{r}
E(G)=\left\{v v_{i j}: 1 \leq i \leq m, 1 \leq j \leq 10 n-6\right\} \cup \\
\left\{v_{i j} v_{i j+1}: 1 \leq i \leq r\right. \\
7,1 \leq j \leq 10 n-
\end{array}
$$

7) $\mathrm{U}$

$\left\{v_{i 1} v_{i(10 n-6)}: 1 \leq i \leq m\right\}$

$|V(G)|=m(10 n-6)+$

Also,

$|E(G)|=2 m(10 n-6)$. Define a bijective function

$f: V(G) \cup E(G) \rightarrow$

6) +1$\}$

by $f(v)=1$,

$\{1,2, \ldots, 3 m(10 n-$

$f\left(v_{i j}\right)=$

(3(10n-6)(i-1)+3j; $j=1,3,5, \ldots, 10 n-7$

$\left\{3(10 n-6)(i-1)+3 j-1 ; \quad j=2,4,6, \ldots, 10 n-f\left(v v_{i j}\right)=\right.$

$V(G)=\left\{v, v_{i j}: 1 \leq i \leq\right.$
$E(G)=\left\{v v_{i j}: 1 \leq i \leq\right.$
$10 n-2\} \cup\left\{v_{i j} v_{i j+1}:\right.$
$3\} \cup\left\{v_{i 1} v_{i(10 n-2)}: 1 \leq\right.$
. Also, $|V(G)|=m(10 n$
and $|E(G)|=2 m(10 n$
Define a bijective function
$f: V(G) \cup E(G) \rightarrow\{1,2$
$f(v)=1$,
$f\left(v_{i j}\right)=$
$\left\{\begin{array}{l}3(10 n-2)(i-1)+3 j \\ 3(10 n-2)(i-1)+3 j\end{array}\right.$
7
$f\left(v v_{i j}\right)=$
$\left(\begin{array}{l}10 n-2)(i-1)+3 j-1 ; \\ 3(10 n)(i)\end{array}\right.$

$\left\{\begin{array}{l}3(10 n-2)(i-1)+3 j-1 \\ 3(10 n-2)(i-1)+3 j\end{array}\right.$

$j=1,3,5, \ldots, 10 n-3$

$f\left(v v_{i j}\right)=$

$\{3(10 n-6)(i-1)+3 j-1$;

$\{3(10 n-6)(i-1)+3 j$;

$j=1,3,5, \ldots, 10 n-7$

$\left.j=2,4,6, \ldots, 10 f_{i j(18 n-2)}\right)=3(10 n-2)(i-1)+3 j+1, j=$

Consider the following cases.

Case $1 . m \neq 2(\bmod 5)$

$f\left(v_{i j} v_{i j+1}\right)=3(10 n-6)(i-1)+3 j+1, j=$ $1,2,3, \ldots, 10 n-7$ 
Consider the following cases.

Case $1 . m \neq 4(\bmod 5)$

$f\left(v_{i j} v_{i j+1}\right)=3(10 n-2)(i-1)+3 j+1, j=$

$f\left(v v_{i j}\right)=\left\{\begin{array}{lr}9(i-1)+3 j-1 ; & j=1,3 \\ 9(i-1)+3 j & j=2\end{array}\right.$

$1,2,3, \ldots, 10 n-3$

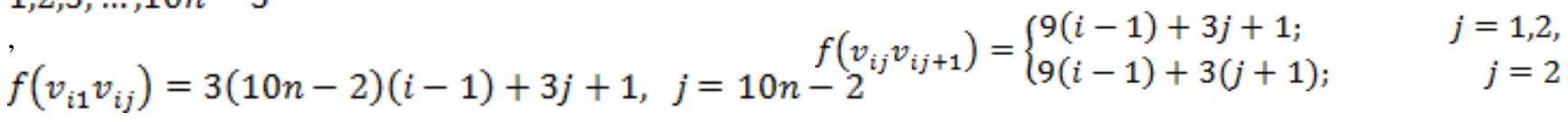

Case $2 . m \equiv 4(\bmod 5)$

$f\left(v_{i j} v_{i j+1}\right)=$

$3(10 n-2)(i-1)+3 j+1$;

$\{3(10 n-2)(i-1)+3(j+1)-1$;

$f\left(v_{i 1} v_{i j}\right)=9(i-1)+3 j+1, j=3$.

$f\left(v_{i 1} v_{i j}\right)=3(10 n-2)(i-1)+3(j-1)+$

1, $j=10 n-2$

Clearly, for any edge $u v \in E(G)$ $j=1,2,3$, It is easily ve 4 ified, for any edge $u v \in E(G)$, the numbers $j=2,4,6, \ldots, 10 n(v)_{3}$ and $f(u v)$ are pairwise relatively prime. Hence $G=W_{3}^{m}$ admits an edge vertex prime graph. $\operatorname{gcd}(f(u), f(v))=1, \operatorname{gcd}(f(u), f(u v))=1$, $\operatorname{gcd}(f(v), f(u v))=1$. Hence $G=W_{10 n-2}^{m}$ $m \geq 1$ and $n \geq 1$ admits an edge vertex prime graph.

Theorem 3.4 One point union of $m$ copies $W_{3}$ is an edge vertex prime graph.

Proof. Let $G=W_{3}^{m}$ be a graph. Then $V(G)=\left\{v, v_{i j}: 1 \leq i \leq m, 1 \leq j \leq 3\right\} \quad$ and $E(G)=\left\{v v_{i j}: 1 \leq i \leq m, 1 \leq j \leq 3\right\} \quad \mathrm{U}$

$\left\{v_{i j} v_{i j+1}: 1 \leq i \leq m, 1 \leq j \leq 2\right\} \cup$

$\left\{v_{i 1} v_{i 3}: 1 \leq i \leq m\right\}$

. Also, $|V(G)|=3 m+1$ and $|E(G)|=6 m$.

Theorem 3.5 One point union of $m$ copies $W_{5}$ is an edge vertex prime graph.

Proof. Let $G=W_{5}^{m}$ be a graph. Then $V(G)=\left\{v, v_{i j}: 1 \leq i \leq m, 1 \leq j \leq 5\right\}$ and $E(G)=\left\{v v_{i j}: 1 \leq i \leq m, 1 \leq j \leq 5\right\}$

$\mathrm{U}$

$\left\{v_{i j} v_{i j+1}: 1 \leq i \leq m, 1 \leq j \leq 4\right\} \cup$

$$
\left\{v_{i 1} v_{i 5}: 1 \leq i \leq m\right\}
$$

. Also, $|V(G)|=5 m+1$ and $|E(G)|=10 m$.

Define a bijective function $f: V(G) \cup E(G) \rightarrow\{1,2, \ldots, 15 m+1\} \quad$ by $f(v)=1$. Consider $i^{\text {th }}$ copy of the following cases. Case 1. Even number of copies, that is, $i=2,4,6, \ldots$ $f\left(v_{i j}\right)=\left\{\begin{array}{lr}15(i-1)+3 j-1 ; & j=1,3,5 \\ 15(i-1)+3 j & j=2,4\end{array}\right.$ Define a bijective function $f: V(G) \cup E(G) \rightarrow\{1,2, \ldots, 9 m+1\}$ by $f(v)=1$. Consider $i^{\text {th }}$ copy of the following cases.

$f\left(v v_{i j}\right)=\left\{\begin{array}{lr}15(i-1)+3 j ; & j=1,3,5 \\ 15(i-1)+3 j-1 ; & j=2,4\end{array}\right.$

Case 1. Even number of copies, that is, $i=2,4,6, \ldots$

$f\left(v_{i j}\right)=\left\{\begin{array}{lr}9(i-1)+3 j-1 ; & j=1,3 \\ 9(i-1)+3 j & j=2\end{array}\right.$

$f\left(v_{i j} v_{i j+1}\right)=15(i-1)+3 j+1, j=1,2,3,4$.

$f\left(v v_{i j}\right)=\left\{\begin{array}{lr}9(i-1)+3 j ; & j=1,3 \\ 9(i-1)+3 j-1 ; & j=2\end{array}\right.$

$f\left(v_{i 1} v_{i j}\right)=15(i-1)+3 j+1, j=5$.

Case 2. Odd number of copies, that is, $i=1,3,5, \ldots$

$f\left(v_{i j} v_{i j+1}\right)=9(i-1)+3 j+1, j=1,2$

$f\left(v_{i j}\right)=\left\{\begin{array}{lr}15(i-1+3 j) & j=1,3 \\ 15(i-1)+3 j-1 ; & j=2,4 \\ 15(i-1)+3 j-2 ; & j=5\end{array}\right.$

$f\left(v_{i 1} v_{i j}\right)=9(i-1)+3 j+1, j=3$.

Case 2. Odd number of copies, that is, $i=1,3,5, \ldots$

$f\left(v_{i j}\right)= \begin{cases}9(i-1+3 j ;) ; & j=1 \\ 9(i-1)+3 j-1 ; & j=2 \\ 9(i-1)+3 j-2 ; & j=3\end{cases}$

$$
\begin{aligned}
& f\left(v v_{i j}\right)=\left\{\begin{array}{lr}
15(i-1)+3 j-1 ; & j=1,3,5 \\
15(i-1)+3 j & j=2,4
\end{array}\right. \\
& f\left(v_{i j} v_{i j+1}\right)=\left\{\begin{array}{cc}
15(i-1)+3 j+1 ; & j=1,2,3 \\
15(i-1)+3 j+3 ; & j=4
\end{array}\right.
\end{aligned}
$$


$f\left(v_{i 1} v_{i j}\right)=15(i-1)+3 j+1, j=5$.

Therefore, for any edge $u v \in E(G)$ $\operatorname{gcd}(f(u), f(v))=1, \operatorname{gcd}(f(u), f(u v))=1$, $\operatorname{gcd}(f(v), f(u v))=1$. Hence $G=W_{5}^{m}$ admits an edge vertex prime graph.

Theorem 3.6 One point union of $m$ copies $W_{7}$ is an edge vertex prime graph.

Proof. Let $G=W_{7}^{m}$ be a graph. Then $V(G)=\left\{v, v_{i j}: 1 \leq i \leq m, 1 \leq j \leq 7\right\}$ and

$$
E(G)=\left\{v v_{i j}: 1 \leq i \leq m, 1 \leq j \leq 7\right\}
$$

$\left\{v_{i j} v_{i j+1}: 1 \leq i \leq m, 1 \leq j \leq 6\right\} \cup$

$\left\{v_{i 1} v_{i 7}: 1 \leq i \leq m\right\}$

. Also, $|V(G)|=7 m+1$ and $|E(G)|=14 m$.

Define bijective

function

$f: V(G) \cup E(G) \rightarrow\{1,2, \ldots, 21 m+1\}$

by

$f(v)=1$. Consider $i^{\text {th }}$ copy of the following cases.

Case 1. Even number of copies, that is, $i=2,4,6, \ldots$

$f\left(v_{i j}\right)=\left\{\begin{array}{lr}21(i-1)+3 j-1 ; & j=1,3,5,7 \\ 21(i-1)+3 j & j=2,4,6\end{array}\right.$

$f\left(v v_{i j}\right)=\left\{\begin{array}{lr}21(i-1)+3 j ; & j=1,3,5,7 \\ 21(i-1)+3 j-1 ; & j=2,4,6\end{array}\right.$

Subcase 1a. $m \neq 4(\bmod 10)$

$f\left(v_{i j} v_{i j+1}\right)=21(i-1)+3 j+1, j=1,2,3,4,5,6$

$f\left(v_{i 1} v_{i j}\right)=21(i-1)+3(j+1)+1, j=7$.

Subcase 1 b. $m \equiv 4(\bmod 10)$

$f\left(v_{i j} v_{i j+1}\right)=21(i-1)+3 j+1, j=1,2,3,4,5$

$f\left(v_{i j} v_{i j+1}\right)=21(i-1)+3(j+1)+1, j=6$

$f\left(v_{i 1} v_{i j}\right)=21(i-1)+3 j-2, j=7$.

Case 2. Odd number of copies, that is, $i=1,3,5, \ldots$

$f\left(v_{i j}\right)=\left\{\begin{array}{lr}21(i-1)+3 j ; & j=1,3,5 \\ 21(i-1)+3 j-1 ; & j=2,4,6 \\ 21(i-1)+3 j-2 ; & j=7\end{array}\right.$

$f\left(v v_{i j}\right)=\left\{\begin{array}{lr}21(i-1)+3 j-1 ; & j=1,3,5,7 \\ 21(i-1)+3 j & j=2,4,6\end{array}\right.$

$f\left(v_{i j} v_{i j+1}\right)=21(i-1)+3 j+1, j=1,2,3,4,5$

$f\left(v_{i 1} v_{i j}\right)=21(i-1)+3 j+3, j=6$.

$$
f\left(v_{i 1} v_{i 7}\right)=21(i-1)+3 j+1, j=7
$$

Clearly, for any edge $u v \in E(G)$, the numbers $f(u), f(v)$ and $f(u v)$ are pairwise

relatively prime. Hence $G=W_{7}^{m}$ admits an edge vertex prime graph.

Theorem 3.7 One point union of $m$ copies $W_{9}$ is an edge vertex prime graph.

Proof. Let $G=W_{9}^{m}$ be a graph. Then $V(G)=\left\{v, v_{i j}: 1 \leq i \leq m, 1 \leq j \leq 9\right\}$ and

$$
E(G)=\left\{v v_{i j}: 1 \leq i \leq m, 1 \leq j \leq 9\right\} \quad u
$$

$\left\{v_{i j} v_{i j+1}: 1 \leq i \leq m, 1 \leq j \leq 8\right\} \cup$

$$
\left\{v_{i 1} v_{i g}: 1 \leq i \leq m\right\}
$$

. Also, $|V(G)|=9 m+1$ and $|E(G)|=18 m$.

Define a bijective function $f: V(G) \cup E(G) \rightarrow\{1,2, \ldots, 27 m+1\} \quad$ by $f(v)=1$. Consider $i^{\text {th }}$ copy of the following cases.

Case 1. Even number of copies, that is, $i=2,4,6, \ldots$

$$
\begin{aligned}
& f\left(v_{i j}\right)=\left\{\begin{array}{lr}
27(i-1)+3 j-1 ; & j=1,3,5,7,9 \\
27(i-1)+3 j & j=2,4,6,8
\end{array}\right. \\
& f\left(v v_{i j}\right)=\left\{\begin{array}{lr}
27(i-1)+3 j ; & j=1,3,5,7,9 \\
27(i-1)+3 j-1 ; & j=2,4,6,8
\end{array}\right. \\
& f\left(v_{i j} v_{i j+1}\right)=27(i-1)+3 j+1, j=1,2,3,4,5,6,7,8
\end{aligned}
$$

Case 2. Odd number of copies, that is, $i=1,3,5, \ldots$

$f\left(v_{i j}\right)=\left\{\begin{array}{lr}27(i-1)+3 j ; & j=1,3,5,7 \\ 27(i-1)+3 j-1 ; & j=2,4,6,8 \\ 27(i-1)+3 j-2 ; & j=9\end{array}\right.$

$f\left(v v_{i j}\right)= \begin{cases}27(i-1)+3 j-1 ; & j=1,3,5,7 \\ 27(i-1)+3 j & j=2,4,6,8\end{cases}$

$f\left(v_{i j} v_{i j+1}\right)=27(i-1)+3 j+1, j=$ $1,2,3,4,5,6,7$

Subcase 2 a. $m \neq 7(\bmod 10)$

$f\left(v v_{i j}\right)=27(i-1)+3 j-1, j=9$,

$f\left(v_{i j} v_{i j+1}\right)=27(i-1)+3 j+1, j=8$,

$f\left(v_{i 1} v_{i j}\right)=27(i-1)+3 j-2, j=9$.

Subcase $2 \mathrm{~b} . m \equiv 7(\bmod 10)$

$f\left(v v_{i j+1}\right)=27(i-1)+3 j+1, j=9$

$f\left(v_{i j} v_{i j+1}\right)=27(i-1)+3(j+1), j=8$

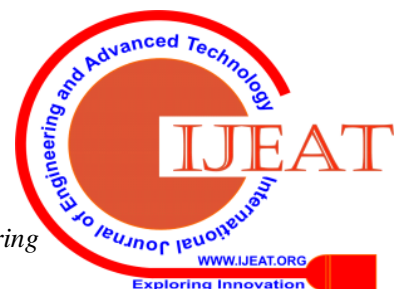




$$
f\left(v_{i 1} v_{i j}\right)=27(i-1)+3 j-1, j=9 .
$$

Clearly, for any edge $u v \in E(G)$, the numbers $f(u), f(v)$ and $f(u v)$ are pairwise relatively prime. Hence $G=W_{9}^{m}$ admits an edge vertex prime graph.

Theorem 3.8 One point union of $m$ copies of $C_{n}^{m}$, $n=3,5,7,9,11$ is an edge vertex

prime graph.

Proof. Let $G=C_{n}^{m},(n=3,5,7,9,11)$ be a graph. Then $V(G)=\left\{v, v_{i j}: 1 \leq i \leq m, 1 \leq j \leq n-1\right\}$ and $\quad E(G)=\left\{v v_{i 1}, v v_{i(n-1)}: 1 \leq i \leq m\right\} \cup$ $\left\{v_{i j} v_{i j+1}: 1 \leq i \leq m, 1 \leq j \leq n-2\right\} \quad$. Also, $|V(G)|=m(n-1)+1$ and $|E(G)|=m n$.

Define a bijective function $f: V(G) \cup E(G) \rightarrow\{1,2, \ldots, 2 m n-m+1\} \quad$ by $f(v)=1$. Consider $i^{\text {th }}$ copy of the following cases. Case 1. Odd number of copies, that is, $i=1,3,5, \ldots$
Define a bijective function $f: V(G) \cup E(G) \rightarrow\{1,2, \ldots, 7 m+1\}$ by $f(v)=1$ Consider $i^{\text {th }}$ copy of the following cases.

Case 1. Odd number of copies, that is, $i=1,3,5, \ldots$ $f\left(v_{i j}\right)=8(i-1)+2(j+1)-i, j=1,2,3$

$f\left(v_{i j} v_{i j+1}\right)=8(i-1)+2(j+2)-(i+1), j=1,2$ $f\left(v v_{i 1}\right)=7 i-5, f\left(v v_{i 3}\right)=7(i+1)+1$.

Case 2. Even number of copies, that is, $i=2,4,6, \ldots$ $f\left(v_{i j}\right)=8(i-1)+2(j+1)-(i+1), j=1,2,3$ $f\left(v_{i j} v_{i j+1}\right)=8(i-1)+2(j+2)-(i+2), j=$ 1

, 2

$f\left(v v_{i 1}\right)=7(i-1)+1, f\left(v v_{i 3}\right)=7 i$.

Therefore, for any edge $u v \in E(G)$, the numbers $f(u), f(v)$ and $f(u v)$ are pairwise relatively prime. Hence $G=C_{4}^{m}$ admits an edge vertex prime graph.

$f\left(v_{i j}\right)=2 n(i-1)+2(j+1)-i, j=1,2,3, \ldots, n$ Theprem 3.10 One point union of $m$ copies of $C_{6}$ is an edge $f\left(v_{i j} v_{i j+1}\right)=2 n(i-1)+2(j+2)-(i+1), j=1,2,3, \ldots, n$, praph.

$f\left(v v_{i 1}\right)=(2 n-1) i-(2 n-3)$,

$f\left(v v_{i(n-1)}\right)=(2 n-1) i+1$.

Case 2. Even number of copies, that is $i=2,4,6, \ldots$ $f\left(v_{i j}\right)=2 n(i-1)+2(j+1)-(i+1), j=1,2,3, \ldots, n-1$. $f\left(v_{i j} v_{i j+1}\right)=2 n(i-1)+2(j+2)-(i+2), j=1,2,3, \ldots n-2$.

Consider the following subcases.

Subcase 2a. Consider $n=3,5,9$, if we take $n=7$, then $m \neq 2(\bmod 6)$ and if we take $n=11$, then $m \neq 4(\bmod 10)$.

$f\left(v v_{i 1}\right)=(2 n-1) i+1$,

$f\left(v v_{i(n-1)}\right)=(2 n-1) i$.

Subcase $2 \mathrm{~b}$. If we take $n=7$, then $m \equiv 2(\bmod 6)$ and if we take $n=11$, then $m \equiv 4(\bmod 10)$.

$f\left(v v_{i 1}\right)=(2 n-1) i_{,}$

$f\left(v v_{i(n-1)}\right)=(2 n-1) i+1$.

Clearly, for any edge $u v \in E(G)$, $\operatorname{gcd}(f(u), f(v))=1, \operatorname{gcd}(f(u), f(u v))=1$, $\operatorname{gcd}(f(v), f(u v))=1$. Hence $G=C_{n}^{m}$

admits an edge vertex prime graph.

Theorem 3.9 One point union of $m$ copies of $C_{4}$ is an edge vertex prime graph.

Proof. Let $G=C_{4}^{m}$ be a graph. Then $V(G)=\left\{v, v_{i j}: 1 \leq i \leq m, 1 \leq j \leq 3\right\}$ and

$E(G)=\left\{v v_{i 1}, v v_{i 3}: 1 \leq i \leq m\right\} \cup$

$\left\{v_{i j} v_{i j+1}: 1 \leq i \leq m, 1 \leq j \leq 2\right\}$

Also, $|V(G)|=3 m+1$ and $|E(G)|=4 m$.

Proof. ${ }^{\text {Let }} G=C_{6}^{m}$ be a graph. Then

$V(G)=\left\{v, v_{i j}: 1 \leq i \leq m, 1 \leq j \leq 5\right\}$ and

$E(G)=\left\{v v_{i 1}, v v_{i 5}: 1 \leq i \leq m\right\} \cup$

$\left\{v_{i j} v_{i j+1}: 1 \leq i \leq m, 1 \leq j \leq 4\right\}$

Also, $|V(G)|=5 m+1$ and $|E(G)|=6 m$.

Define a bijective function $f: V(G) \cup E(G) \rightarrow\{1,2, \ldots, 11 m+1\} \quad$ by $f(v)=1$. Consider $i^{\text {th }}$ copy of the following cases.

Case 1. Odd number of copies, that is, $i=1,3,5, \ldots$

$f\left(v_{i j}\right)=12(i-1)+2(j+1)-i, j=1,2,3,4,5$

$f\left(v_{i j} v_{i j+1}\right)=12(i-1)+2(j+2)-(i+1), j=1,2,3,4$

$f\left(v v_{i 1}\right)=11 i-9, f\left(v v_{i 5}\right)=11 i+1$.

Case 2. Even number of copies, that is, $i=2,4,6, \ldots$

$f\left(v_{i j}\right)=12(i-1)+2(j+1)-(i+1), j=1,2,3,4,5$

$f\left(v_{i j} v_{i j+1}\right)=12(i-1)+2(j+2)-(i+$

2), $j=1$

$, 2,3,4$

Subcase 2a. $m \not 4(\bmod 10)$

$f\left(v v_{i 1}\right)=11 i+1, f\left(v v_{i 5}\right)=11 i$.

Subcase 2 b. $m \equiv 4(\bmod 10)$

$f\left(v v_{i 1}\right)=11 i, f\left(v v_{i 5}\right)=11 i+1$

Therefore, for any edge $u v \in E(G)$ $\operatorname{gcd}(f(u), f(v))=1, \operatorname{gcd}(f(u), f(u v))=1$, $\operatorname{gcd}(f(v), f(u v))=1$. Hence $G=C_{6}^{m}$ admits an edge vertex prime graph.

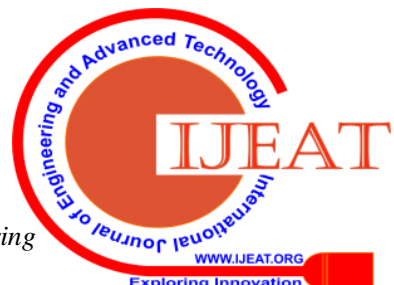




\section{CONCLUSION}

We proved that if $G(p, q)$ has an edge vertex prime graph with $p+q$ is even, then there exists a graph from the class $G \cup P_{n}$ that admits an edge vertex prime graph. One point union of $m$ copies $W_{5}$ is an edge vertex prime graph. One point union of $m$ copies $W_{7}$ is an edge vertex prime graph. One point union of $m$ copies $W_{9}$ is an edge vertex prime graph. One point union of $m$ copies of $C_{n}^{m}$, $n=3,5,7,9,11$ is an edge vertex . One point union of $m$ copies of $C_{6}$ is an edge vertex prime graph.

\section{REFERENCES}

1. Balakrishnan. R and Ranganathan. K, A Text Book of Graph Theory, Second Edition, Springer. New York 2012.

2. Gallian. J. A, A Dynamic Survey of Graph Labeling, Electronic Journal of Combinatorics, DS6, 2015.

3. Jagadesh. R, Baskar Babujee. J, Edge Vertex Prime Labeling for some class of Graphs, National Conference on Recent Trends in Mathematics and its Applications, 24-25 February 2017, SRM University, Vadapalani, Chennai, India.

4. Jagadesh. R, Baskar Babujee. J, On Edge Vertex Prime Labeling, International Journal of Pure and Applied Mathematics, Vol.114 No.6, 2017, 209-218.

5. Parmar. Y, Edge Vertex Prime Labeling for Wheel, Fan and Friendship Graph, International Journal of Mathematics and Statistics Invention, Vol.5 Issue 8, October 2017, 23-29.

6. Parmar. Y, Vertex Prime Labeling for $K_{2, m}$ and $K_{3, m}$ Graphs, Mathematical Journal of Interdisciplinary Sciences Vol.6 No.2, March 2018, 167-180.

7. Simaringa. M, Muthukumaran. S, Edge Vertex Prime Labeling of Some Graphs, Malaya Journal of Mathematik, Vol.7, No.2, 264-268, 2019.

8. Simaringa. M, Muthukumaran. S, Subhasri.S, Edge Vertex Prime Labeling of Trees, JETIR,Vol.6, Issue.5, 282-287, May-2019.

9. Simaringa. M, Muthukumaran. S, Edge Vertex Prime Labeling of Graphs, Malaya Journal of Mathematik, Vol.7, No.3, 572-578, 2019.

10. Tout. A, Dabboucy. A. N, Howalla. K, Prime Labeling of Graphs, National Academy Science, Letters, 11,1982, 365-368. 